\title{
Diagnostic ability of the fetal ultrasonographic parameters in screening for gestational diabetes
}

\begin{abstract}
Pancreatic $\beta$-cell hyperplasia occurring in normal pregnancy results in higher fasting and postprandial insulin levels. In addition, placental hormones cause increased insulin resistance, especially throughout the third trimester. Pregnancies with GDM are associated with increased obstetric complications. Therefore, the surveillance of GDM during pregnancy is especially important. The best method for screening continues to be controversial. O' Sullivan, is the most widely used screening test and is recommended by the ADA. GDM of any severity increases the risk of fetal macrosomia which can be diagnosed by ultrasonography Increased body weight is due to organomegaly and increased fat deposition. Increased glucose transfer from the diabetic mother to the fetus and placenta results in fetal hyperglycemia and hyperinsulinemia, promoting growth of insulin-dependent tissues and organs, such as the liver. The aim of the present study is to test the efficiency of ultrasound parameters (biparietal diameter, abdominal circumference, estimated fetal weight, fetal truncal subcutaneous fat layer, and fetal liver length) against the efficiency of the 50gram oral glucose challenge test ( $\mathrm{O}^{\prime}$ Sullivan test) to screen for gestational diabetes in the second trimester between 24-28weeks of gestation.

The study enrolled three hundred second trimester pregnant subjects selected from outpatient clinic coming for antenatal visit at 24-28weeks gestation. All recruited patients were subjected to history taking, complete clinical examination, ultrasound examination(Including: fetal biometry measurements to confirm gestational age, detailed anomaly scan, fetal subcutaneous fat layer, length of the right lobe of the liver, abdominal circumference, biparietal diameter, head circumference, femur length, and estimated fetal weight calculated by (Hadlock AC,BPD) in grams O'Sullivan test was done, patients were considered screen positive if plasma venous glucose concentration $\geq 140 \mathrm{md} / \mathrm{dl}$ or $7.8 \mathrm{mmol} / \mathrm{L} .100$ gram oral glucose tolerance curve was used to confirm or rule out the diagnosis of gestational diabetes. A blood glucose level below $180 \mathrm{mg}$ after one hour was considered normal. A blood glucose level below $140 \mathrm{mg}$ after two hours is considered normal. Two or more abnormally high readings are considered Diabetes.
\end{abstract}

Volume 6 Issue I - 2017

\author{
Eman Ali Abd El Fattah \\ Department of Obstetrics and Gynecology,Alexandria \\ University, Shatby Maternity hospital, Egypt
}

Correspondence: Eman Ali Abd El Fattah, Department of Obstetrics and Gynecology, Faculty of Medicine, Alexandria University, Shatby Maternity hospital, 4, fathia Baheeg St, Fleming, Alexandria, Egypt, Email eman0eman0eman7@Gmail.com

Received: February II, 2017 | Published: October 27, 2017

\section{Introduction}

Gestational diabetes mellitus (GDM) is a condition of glucose intolerance with onset in pregnancy 1,2) It is associated with increased obstetric complications, such as fetal macrosomia, neonatal hypoglycemia and hypocalcemia, as well as maternal hypertension and thromboembolic disease. Therefore, surveillance for GDM is important. ${ }^{1,2}$ Women at risk should be tested at the first antenatal visit using the American Diabetes Association diagnostic criteria for non- pregnant adults. ${ }^{2}$ The 50 -g non-fasting 1-h glucose challenge test (GCT) is the most widely implemented screen used. Alternative screening methods have been proposed to increase the detection rates of GDM and to overcome these shortcomings of the GCT. Some are based on ultrasound examinations. Although uncomplicated GDM with less severe fasting hyperglycemia has not been associated with increased perinatal mortality, GDM of any severity increases the risk of fetal macrosomia. ${ }^{3}$ Increased glucose transfer from the diabetic mother to the fetus and placenta results in fetal hyperglycemia and hyperinsulinemia, promoting growth of insulin-dependent tissues and organs, such as the liver. ${ }^{4}$ The aim of the present study is to test the efficiency of ultrasound parameters (biparietal diameter, abdominal circumference, estimated fetal weight, fetal truncal subcutaneous fat layer, and fetal liver length) against the efficiency of the 50 gram oral glucose challenge test (O' Sullivan test) to screen for gestational diabetes in the second trimester between $24-28$ weeks of gestation.

\section{Methods}

The study enrolled three hundred second trimester pregnant subjects selected from outpatient clinic coming for antenatal visit at 24-28weeks gestation, with or without history of gestational diabetes in a previous pregnancy. Subjects with pre-gestational diabetes were excluded. Consent was taken from all subjects to participate in the study. All recruited patients were subjected to history taking, complete clinical examination, ultrasound examination(Including: fetal biometry measurements to confirm gestational age, detailed anomaly scan, fetal subcutaneous fat layer, length of the right lobe of the liver, abdominal circumference, biparietal diameter, head circumference, femur length, and estimated fetal weight calculated by (Hadlock AC,BPD) in grams. All examinations were performed by the same sonologist (E.A) using an HDI 1500 scanner equipped with a 3.5-MHz transducer (Medison ultrasound, SonoR-7). To measure the fetal anterior abdominal wall fat, the abdominal circumference was selected. The measurement was taken as close to vertical as possible. The quadrant that included the spine was avoided. The measurements were taken from the inner to the outer aspect of the echogenic subcutaneous fat that surrounded 
the abdomen. Patient is considered screen positive if expected fetal weight or abdominal circumference is above the $90^{\text {th }}$ percentile for age, or measured fat layer equals to or exceeds $3.5 \mathrm{~mm}$ at $24-28$ weeks gestation. To measure liver length, the tip of the right lobe was clearly identified and the liver length was measured from the dome of the right hemi-diaphragm to the tip of the right lobe (Figure 1). O'Sullivan test was done; patients received 50gram glucose orally irrespective of their fasting state. Blood sugar level was evaluated one hour later. Patients were considered screen positive if plasma venous glucose concentration $\geq 140 \mathrm{md} / \mathrm{dl}$ or $7.8 \mathrm{mmol} / \mathrm{L} .{ }^{5}$ Screen positive cases were subjected to 100gram oral glucose tolerance curve: (2hours 100gram OGTT) to confirm or rule out the diagnosis of gestational diabetes. The patient's blood glucose was recorded at baseline after eight hours fasting. The patient was then given 100gram glucose orally then blood glucose was measured at one hour and two hours. The diagnosis of GDM was based on the criteria of the World Health Organization, which is considered the gold standard. A blood glucose level below $180 \mathrm{mg}$ after one hour was considered normal. A blood glucose level below $140 \mathrm{mg}$ after two hours is considered normal. One abnormal reading denotes glucose intolerance and requires further assessment at a later gestation (later than 28weeks). Otherwise; abnormal reading on two occasions of the 100gram OGTT, the patient is diagnosed as having gestational diabetes.

\section{Statistical methodology}

Data were collected and entered to the computer using SPSS (Statistical Package for Social Science) program for statistical analysis (ver 21). ${ }^{5}$ Data were entered as numerical or categorical, as appropriate. When Kolmogorov-Smirnov test revealed no significance in the distribution of variables, parametric statistics was carried out, while in the not-normally distributed data the non-parametric statistics was carried out. ${ }^{6}$

i. Data were described using minimum, maximum, mean, and standard deviation for the normally distributed data.

ii. Data were described using minimum, maximum, median and inter-quartile range for not-normally distributed data.

iii. Comparisons were carried out between two studied independent not-normally distributed subgroups using Mann-Whitney U test. ${ }^{7}$

iv. Histograms with distribution curve, Box and Whiskers graph, bar chart and clustered bar chart were used accordingly.

v. Area under the ROC (AUC) was carried using MedCalc Software version $14 .{ }^{8,9}$

An alpha level was set to $5 \%$ with a significance level of $95 \%$, and a beta error accepted up to $20 \%$ with a power of study of $80 \%$. In Table I \& Figure 2, gestational age was statistically not significant ( $p>0.05$ ). On the other hand a statistically significant difference was found in results of OGTT between cases with positive O' Sullivan test and cases with negative O' Sullivan test $(\mathrm{p}=0.000)$ (Table 2) \& (Figure 3), fetal abdominal circumference $(\mathrm{p}=0.001)$ (Table 3) \& (Figure 4), fetal Biparietal diameter $(\mathrm{p}=0.000)$ (Table 4) \& (Figure 5), Femur length $(\mathrm{p}=0.000)$ (Table 5) and (Figure 6), expected fetal weight $(\mathrm{p}=0.000)$ (Table 6) \& (Figure 7), Abdominal subcutaneous tissue fat $(\mathrm{p}=0.000)$ (Table 7) \& (Figure 8). No statistically significant difference found between cases with positive OGTT and cases with negative OGTT as regards Fetal liver length as $p=0.060$ (Table 8) \& (Figure 9).

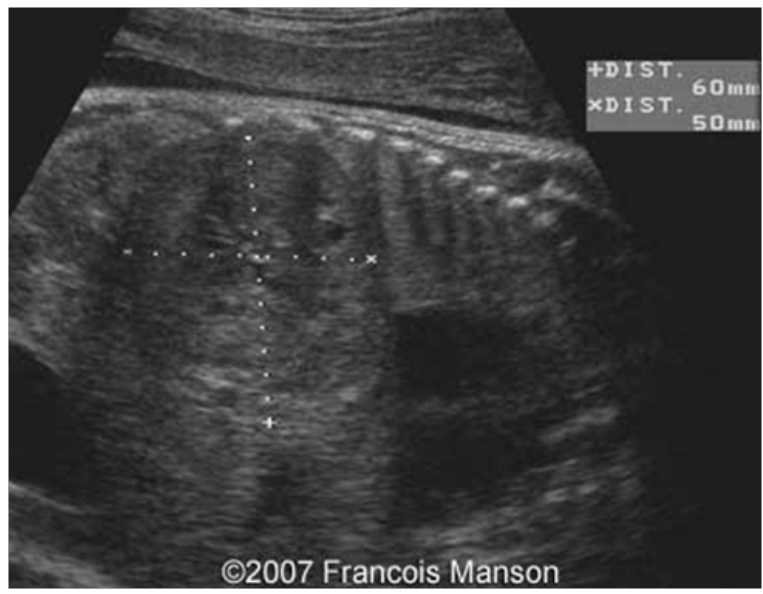

Figure I Fetal live.

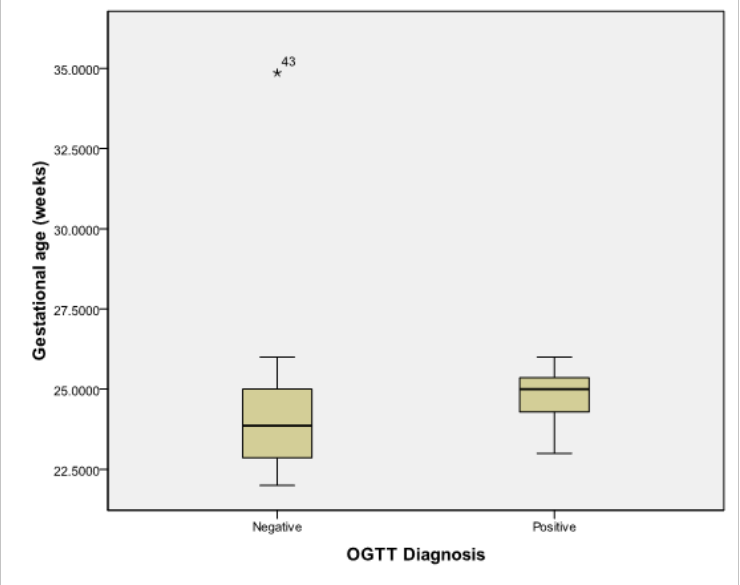

Figure 2 Box and whisker graph of gestational age (weeks), the thick line in the middle of the box represents the median, the box represents the interquartile range (from 25 th to 75 th percentiles), the whiskers represents the minimum and maximum values after excluding outliers (black-filled circle) and extremes.

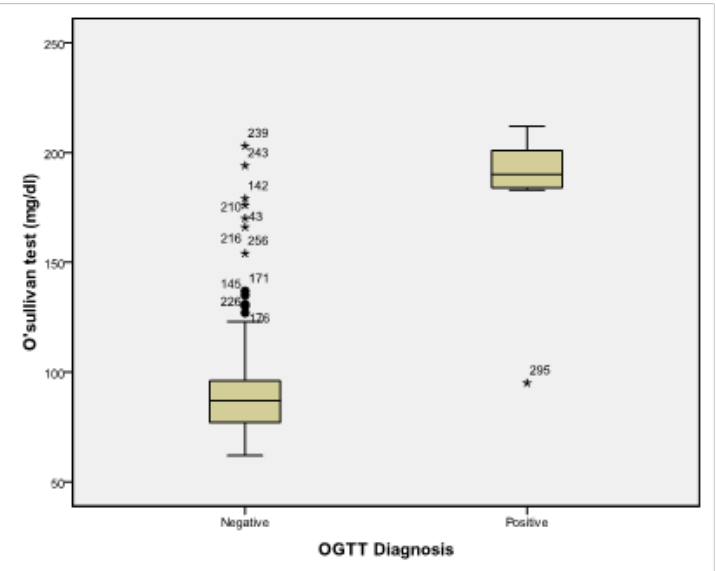

Figure 3 Box and whisker graph of O'sullivan test $(\mathrm{mg} / \mathrm{dl})$, the thick line in the middle of the box represents the median, the box represents the inter-quartile range (from 25 th to 75 th percentiles), the whiskers represents the minimum and maximum values after excluding outliers (black-filled circle) and extremes. 


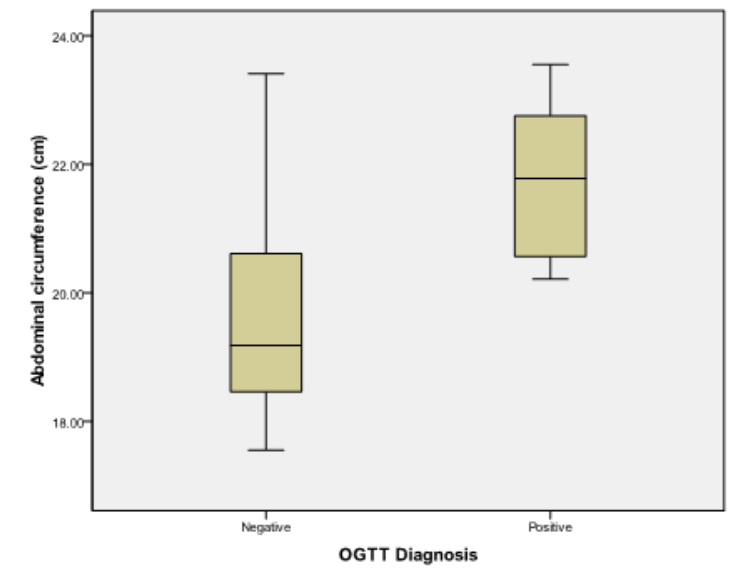

Figure 4 Box and whisker graph of abdominal circumference $(\mathrm{cm})$, the thick line in the middle of the box represents the median, the box represents the inter-quartile range (from 25 th to 75 th percentiles), the whiskers represents the minimum and maximum values after excluding outliers (black-filled circle) and extremes.

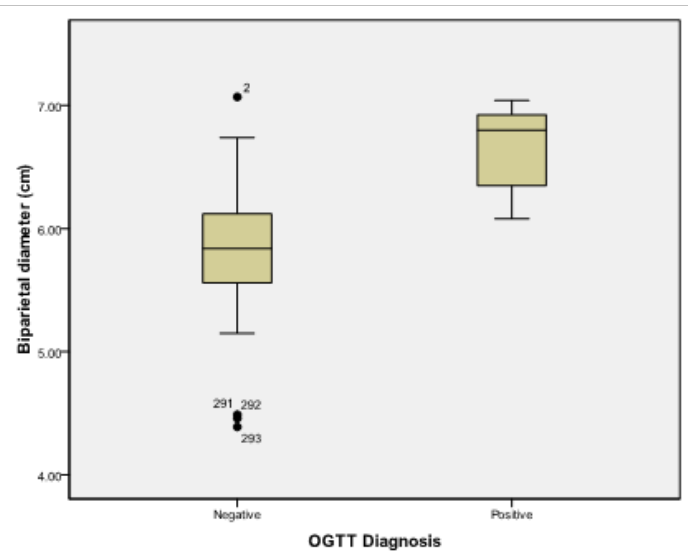

Figure 5 Box and whisker graph of biparietal diameter $(\mathrm{cm})$, the thick line in the middle of the box represents the median, the box represents the interquartile range (from 25 th to 75 th percentiles), the whiskers represents the minimum and maximum values after excluding outliers (black-filled circle) and extremes.

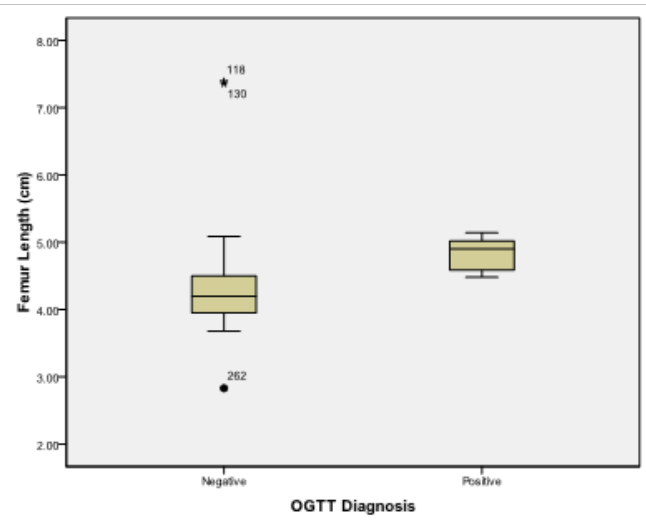

Figure 6 Box and whisker graph of femur length, the thick line in the middle of the box represents the median, the box represents the inter-quartile range (from 25th to 75 th percentiles), the whiskers represents the minimum and maximum values after excluding outliers (black-filled circle) and extremes.

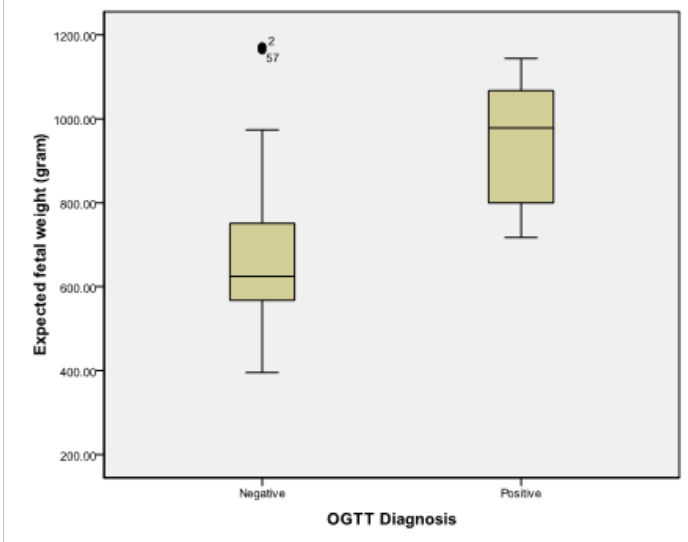

Figure 7 Box and whisker graph of expected fetal weight (gram), the thick line in the middle of the box represents the median, the box represents the inter-quartile range (from 25 th to 75 th percentiles), the whiskers represents the minimum and maximum values after excluding outliers (black-filled circle) and extremes.

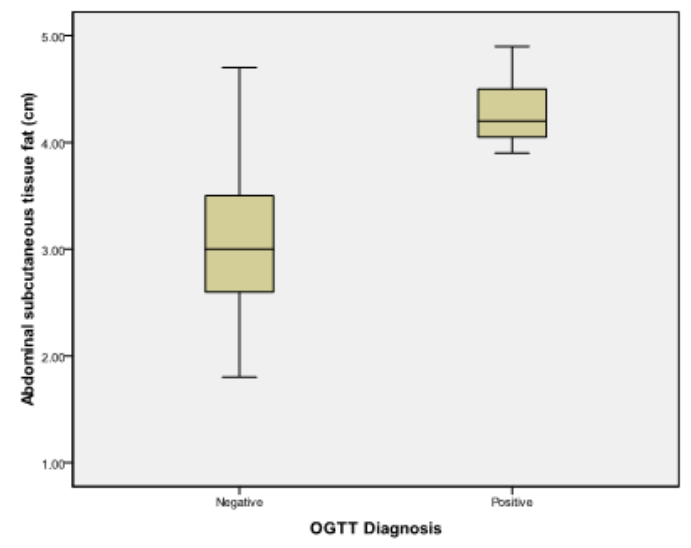

Figure 8 Box and whisker graph of expected abdominal subcutaneous tissue fat $(\mathrm{cm})$, the thick line in the middle of the box represents the median, the box represents the inter-quartile range (from 25th to 75th percentiles), the whiskers represents the minimum and maximum values after excluding outliers (black-filled circle) and extremes.

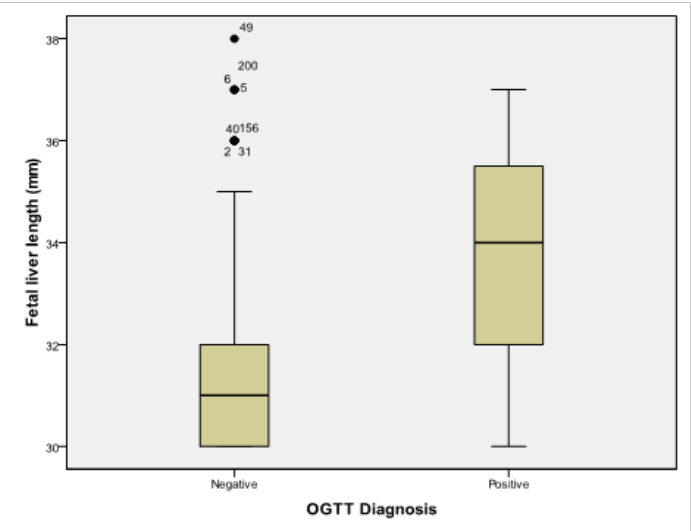

Figure 9 Box and whisker graph of fetal liver length $(\mathrm{mm})$, the thick line in the middle of the box represents the median, the box represents the inter-quartile range (from 25 th to 75 th percentiles), the whiskers represents the minimum and maximum values after excluding outliers (black-filled circle) and extremes. 
Table I Gestational age

\begin{tabular}{llll}
\hline & OGTT negative $(\mathbf{n}=\mathbf{2 9 3})$ & OGTT positive $(\mathbf{n}=\mathbf{7})$ & All cases $(\mathbf{n}=\mathbf{3 0 0})$ \\
\hline Minimum & 22 & 23 & 22 \\
Maximum & 34.86 & 26 & 34.86 \\
Mean & 23.94 & 24.76 & 23.96 \\
Std. Deviation & 1.338 & 1.0192 & 1.336 \\
Median & 23.857 & 25 & 23.857 \\
Inter-quartile range & $22.857-25.000$ & $23.857-25.428$ & $22.857-25.00$ \\
& $\mathrm{D}=0.073$ & $\mathrm{D}=0.198$ & $\mathrm{D}=0.071$ \\
KS test of normality & $\mathrm{p}=0.001 *$ & $\mathrm{p}=0.200 \mathrm{NS}$ & $\mathrm{p}=0.001 *$ \\
& $\mathrm{Z}=1.862, \mathrm{p}=0.063 \mathrm{NS}$ & & \\
\hline
\end{tabular}

i. KS, kolmogorov-smirnov.

ii. *: Statistically not significant $(p<0.05)$,

iii. NS: Statistically not significant $(\mathrm{p}>0.05)$.

Table 2 O'Sullivan test $(\mathrm{mg} / \mathrm{dl})$

\begin{tabular}{llll}
\hline & OGTT negative $(\mathbf{n}=\mathbf{2 9 3})$ & OGTT positive(n=7) & All cases(n=300) \\
\hline Minimum & 62 & 95 & 62 \\
Maximum & 203 & 212 & 212 \\
Mean & 90.15 & 181 & 92.27 \\
Std. Deviation & 19.892 & 39.319 & 24.62 \\
Median & 87 & 190 & 88 \\
Inter-quartile range & $77.00-96.00$ & $183.00-204.00$ & $78.00-96.00$ \\
KS test of normality & $\mathrm{D}=0.152$ & $\mathrm{D}=0.377$ & $\mathrm{D}=0.193$ \\
& $\mathrm{p}=0.000^{*}$ & $\mathrm{p}=0.003^{*}$ & $\mathrm{p}=0.000^{*}$ \\
Mann-Whitney U Test & $\mathrm{Z}=4.124, \mathrm{p}=0.000^{*}$ & &
\end{tabular}

i. KS, kolmogorov-smirnov.

ii. *: Statistically not significant $(p<0.05)$,

Table 3 Abdominal circumference $(\mathrm{cm})$

\begin{tabular}{llll}
\hline & OGTT negative(n=293) & OGTT positive(n=7) & All cases(n=300) \\
\hline Minimum & 17.55 & 20.21 & 17.55 \\
Maximum & 23.41 & 23.55 & 23.55 \\
Mean & 19.4757 & 21.74 & 19.5285 \\
Std. Deviation & 1.17601 & 1.3696 & 1.22699 \\
Median & 19.18 & 21.78 & 19.19 \\
Inter-quartile range & $18.46-20.61$ & $20.25-23.31$ & $18.49-20.71$ \\
KS test of normality & $\mathrm{D}=0.115$ & $\mathrm{D}=0.164$ & $\mathrm{D}=0.117$ \\
& $\mathrm{p}=0.000^{*}$ & $\mathrm{p}=0.200 \mathrm{NS}$ & $\mathrm{p}=0.000^{*}$ \\
\hline
\end{tabular}

i. KS, kolmogorov-smirnov.

ii. $\quad *$ Statistically not significant $(p<0.05)$,

iii. NS, statistically not significant $(p>0.05)$. 
Table 4 Biparietal diameter $(\mathrm{cm})$

\begin{tabular}{llll}
\hline & OGTT negative $(\mathbf{n}=\mathbf{2 9 3})$ & OGTT positive $(\mathbf{n}=7)$ & All cases(n=300) \\
\hline Minimum & 4.39 & 6.08 & 4.39 \\
Maximum & 7.07 & 7.04 & 7.07 \\
Mean & 5.8455 & 6.6386 & 5.864 \\
Std. Deviation & 0.37773 & 0.3812 & 0.39577 \\
Median & 5.84 & 6.8 & 5.855 \\
Inter-quartile range & $5.560-6.125$ & $6.250-7.030$ & $5.5625-6.1500$ \\
KS test of normality & $\mathrm{D}=0.057$ & $\mathrm{D}=0.235$ & $\mathrm{D}=0.060$ \\
Mann-Whitney U Test & $\mathrm{p}=0.022^{*}$ & $\mathrm{p}=0.200 \mathrm{NS}$ & $\mathrm{p}=0.010 *$
\end{tabular}

i. KS, kolmogorov-smirnov.

ii. $\quad$ *: Statistically not significant $(\mathrm{p}<0.05)$,

iii. NS, statistically not significant $(\mathrm{p}>0.05)$.

Table 5 Femur length $(\mathrm{cm})$

\begin{tabular}{llll}
\hline & OGTT negative $(\mathbf{n}=\mathbf{2 9 3})$ & OGTT positive $(\mathbf{n}=\mathbf{7})$ & All cases(n=300) \\
\hline Minimum & 2.83 & 4.48 & 2.83 \\
Maximum & 7.39 & 5.14 & 7.39 \\
Mean & 4.2494 & 4.8186 & 4.2627 \\
Std. Deviation & 0.42555 & 0.26959 & 0.43095 \\
Median & 4.2 & 4.9 & 4.22 \\
Inter-quartile range & $3.95-4.50$ & $4.52-5.10$ & $3.9525-4.5175$ \\
& $\mathrm{D}=0.093$ & $\mathrm{D}=0.190$ & $\mathrm{D}=0.091$ \\
KS test of normality & $\mathrm{p}=0.000^{*}$ & $\mathrm{p}=0.200 \mathrm{NS}$ & $\mathrm{p}=0.000^{*}$ \\
& $\mathrm{Z}=3.635, \mathrm{p}=0.000^{*}$ & &
\end{tabular}

i. KS, kolmogorov-smirnov.

ii. *: Statistically not significant $(\mathrm{p}<0.05)$,

iii. NS, statistically not significant $(\mathrm{p}>0.05)$.

Table 6 Expected fetal weight (gram)

\begin{tabular}{llll}
\hline & OGTT negative $(\mathbf{n}=\mathbf{2 9 3})$ & OGTT positive(n=7) & All cases(n=300) \\
\hline Minimum & 395 & 717 & 395 \\
Maximum & 1172 & 1144 & 1172 \\
Mean & 661.2321 & 938.8571 & 667.71 \\
Std. Deviation & 118.74135 & 171.47247 & 126.97159 \\
Median & 625 & 978 & 631.5 \\
Inter-quartile range & $568.00-751.00$ & $755.00-1130.00$ & $568.25-759.00$ \\
KS test of normality & $\mathrm{D}=0.122$ & $\mathrm{D}=0.162$ & $\mathrm{D}=0.122$ \\
& $\mathrm{p}=0.000^{*}$ & $\mathrm{p}=0.200 \mathrm{NS}$ & $\mathrm{p}=0.000^{*}$ \\
\hline
\end{tabular}

\footnotetext{
i. KS, kolmogorov-smirnov.

ii. $\quad *$ : Statistically not significant $(\mathrm{p}<0.05)$,

iii. NS, statistically not significant $(p>0.05)$
} 
Table 7 Abdominal subcutaneous tissue fat $(\mathrm{cm})$

\begin{tabular}{llll}
\hline & OGTT negative $(\mathbf{n}=\mathbf{2 9 3})$ & OGTT positive $(\mathbf{n}=7)$ & All cases $(\mathbf{n}=\mathbf{3 0 0})$ \\
\hline Minimum & 1.8 & 3.9 & 1.8 \\
Maximum & 4.7 & 4.9 & 4.9 \\
Mean & 3.0567 & 4.3 & 3.0857 \\
Std. Deviation & 0.56314 & 0.35119 & 0.58951 \\
Median & 3 & 4.2 & 3.05 \\
Inter-quartile range & $2.60-3.50$ & $4.00-4.50$ & $2.60-3.50$ \\
KS test of normality & $\mathrm{D}=0.084$ & $\mathrm{D}=0.184$ & $\mathrm{D}=0.073$ \\
& $\mathrm{p}=0.000^{*}$ & $\mathrm{p}=0.200 \mathrm{NS}$ & $\mathrm{p}=0.001^{*}$ \\
\hline
\end{tabular}

i. KS, kolmogorov-smirnov.

ii. $\quad *$ : Statistically not significant $(p<0.05)$,

iii. NS. statistically not significant $(p>0.05)$.

Table 8 Fetal liver length $(\mathrm{mm})$

\begin{tabular}{llll}
\hline & OGTT negative $(\mathbf{n}=\mathbf{2 9 3})$ & OGTT positive(n=7) & All cases(n=300) \\
\hline Minimum & 30 & 30 & 30 \\
Maximum & 38 & 37 & 38 \\
Mean & 31.78 & 33.71 & 31.83 \\
Std. Deviation & 1.655 & 2.752 & 1.706 \\
Median & 31 & 34 & 31.5 \\
Inter-quartile range & $30.00-32.50$ & $30.00-36.00$ & $30.00-33.00$ \\
KS test of normality & $\mathrm{D}=0.199$ & $\mathrm{D}=0.256$ & $\mathrm{D}=0.200$ \\
& $\mathrm{p}=0.000^{*}$ & $\mathrm{p}=0.185 \mathrm{NS}$ & $\mathrm{p}=0.000^{*}$ \\
Mann-Whitney U Test & $\mathrm{Z}=1.883$ & & \\
\hline
\end{tabular}

i. KS, kolmogorov-smirnov.

ii. $\quad *$ : Statistically not significant $(\mathrm{p}<0.05)$,

iii. NS, statistically not significant $(\mathrm{p}>0.05)$.

Table 9 Abdominal circumference $(\mathrm{cm})$

ROC Curve

\begin{tabular}{ll}
\hline Variable & Abdominal Circumference $(\mathrm{cm})$ \\
Classification Variable & OGTT_Diagnosis \\
Sample size & 300 \\
Positive group: OGTT Diagnosis $=1$ & 7 \\
Negative group: OGTT Diagnosis $=0$ & 293 \\
Disease prevalence $(\%)$ & 2.33 \\
\hline
\end{tabular}


Area under the ROC curve (AUC)

\begin{tabular}{ll}
\hline Area under the ROC curve (AUC) & 0.878 \\
Standard Error $^{\mathrm{a}}$ & 0.0615 \\
$95 \%$ Confidence interval $^{\mathrm{b}}$ & 0.836 to 0.913 \\
$\mathrm{z}$ statistic & 6.148 \\
Significance level P (Area $=0.5)$ & $<0.0001$ \\
\hline
\end{tabular}

${ }^{\mathrm{a} D e L o n g ~ e t ~ a l .8 ~}$

${ }^{\mathrm{b} B i n o m i a l ~ e x a c t ~}$

Youden Index

$\begin{array}{ll}\text { Youden index J } & 0.6587 \\ 95 \% \text { Confidence interval }^{\mathrm{a}} & 0.5714 \text { to } 0.7143 \\ \text { Associated criterion } & >20.2 \\ 95 \% \text { Confidence interval } & >20.12 \text { to }>21.69 \\ \text { Sensitivity } & 100 \\ \text { Specificity } & 65.87\end{array}$

${ }^{\text {a}}$ BCa bootstrap confidence interval (I000 iterations; random number seed: 978$)$.

Table 10 Biparietal diameter $(\mathrm{cm})$

\section{ROC curve}

\begin{tabular}{ll}
\hline Variable & Biparietal diameter $(\mathrm{cm})$ \\
Classification Variable & OGTT Diagnosis \\
Sample Size & 300 \\
Positive group: OGTT Diagnosis $=1$ & 7 \\
Negative group: OGTT Diagnosis $=0$ & 293 \\
Disease prevalence $(\%)$ & 2.33
\end{tabular}

Area under the ROC curve (AUC)

\begin{tabular}{ll}
\hline Area under the ROC curve (AUC) & 0.927 \\
Standard Error $^{\mathrm{a}}$ & 0.0431 \\
$95 \%$ Confidence interval $^{\mathrm{b}}$ & 0.891 to 0.954 \\
Z statistic & 9.894 \\
Significance level P (Area $=0.5)$ & $<0.0001$ \\
\hline
\end{tabular}

aDeLong et al. ${ }^{8}$

bBinomial exact

Youden Index

\begin{tabular}{ll}
\hline Youden index J & 0.7036 \\
$95 \%$ Confidence interval $^{\mathrm{a}}$ & 0.6314 to 0.8055 \\
Associated criterion & $>6.24$ \\
$95 \%$ Confidence interval & \\
Sensitivity & $>6.07$ to $>6.74$ \\
Specificity & 85.71 \\
\hline
\end{tabular}

${ }^{\mathrm{a}} \mathrm{BCa}$ bootstrap confidence interval ( 1000 iterations; random number seed: 978).
Table I I Femur length

\section{ROC Curve}

\begin{tabular}{ll}
\hline Variable & Femur Length $(\mathrm{cm})$ \\
Classification variable & OGTT Diagnosis \\
Sample size & 300 \\
Positive group: OGTT Diagnosis $=1$ & 7 \\
Negative group: OGTT Diagnosis $=0$ & 293 \\
Disease prevalence $(\%)$ & 2.33 \\
\hline
\end{tabular}

Area under the ROC curve (AUC)

\begin{tabular}{ll}
\hline Area under the ROC curve (AUC) & 0.902 \\
Standard Error & \\
$95 \%$ Confidence Interval & \\
& 0.0457 \\
z statistic & 0.863 to 0.933 \\
Significance level P $($ Area $=0.5)$ & 8.795 \\
\hline
\end{tabular}

${ }^{\mathrm{a}}$ DeLong et al. ${ }^{8}$

${ }^{\mathrm{b}}$ Binomial exact

Youden Index

\begin{tabular}{ll} 
Youden Index $\mathrm{J}$ & 0.7235 \\
$95 \%$ Confidence Interval $^{\mathrm{a}}$ & 0.6485 to 0.7816 \\
Associated Criterion & $>4.46$ \\
$95 \%$ Confidence Interval & \\
Sensitivity & $>4.42$ to $>4.51$ \\
Specificity & 100 \\
\hline
\end{tabular}

aBCa bootstrap confidence interval (1000 iterations; random number seed: 978). 
Table 12 Expected fetal weight (gram).

ROC curve

\begin{tabular}{ll}
\hline Variable & Expected fetal weight (gram) \\
Classification Variable & OGTT Diagnosis \\
Sample size & 300 \\
Positive group: OGTT Diagnosis $=1$ & 7 \\
Negative group: OGTT Diagnosis $=0$ & 293 \\
Disease Prevalence $(\%)$ & 2.33 \\
\hline
\end{tabular}

\begin{tabular}{ll}
\hline Area under the ROC curve (AUC) & \\
\cline { 2 - 2 } Area under the ROC curve (AUC) & 0.902 \\
Standard Error & \\
95\% Confidence Interval & \\
z statistic & 0.0511 \\
Significance level P (Area $=0.5)$ & 0.863 to 0.934 \\
\hline
\end{tabular}

a DeLong et al. ${ }^{8}$

${ }^{\mathrm{b} B i n o m i a l}$ exact

Youden Index

\begin{tabular}{ll}
\hline Youden Index $\mathrm{J}$ & 0.6758 \\
$95 \%$ Confidence Interval $^{\mathrm{a}}$ & 0.5904 to 0.7411 \\
Associated Criterion & $>712$ \\
$95 \%$ Confidence Interval $^{\mathrm{a}}$ & $>708$ to $>829$ \\
Sensitivity & 100 \\
Specificity & 67.58 \\
\hline
\end{tabular}

${ }^{\mathrm{a} B C a}$ bootstrap confidence interval (1000 iterations; random number seed: 978).

Table I 3 Abdominal subcutaneous tissue fat $(\mathrm{cm})$

ROC curve

\begin{tabular}{ll}
\hline Variable & $\begin{array}{l}\text { Abdominal subcutaneous tissue } \\
\text { fat }(\mathrm{cm})\end{array}$ \\
$\begin{array}{l}\text { OGTT Diagnosis } \\
\text { Slassification variable }\end{array}$ & 300 \\
$\begin{array}{l}\text { Pample size } \\
\text { Negative group: }\end{array}$ & 7 \\
$\begin{array}{l}\text { OGTT Diagnosis }=0 \\
\text { Disease prevalence }(\%)\end{array}$ & 293 \\
\hline
\end{tabular}

Area under the ROC curve (AUC)

\begin{tabular}{ll}
\hline Area under the ROC curve (AUC) & 0.983 \\
Standard Error $^{\mathrm{a}}$ & 0.00819 \\
$95 \%$ Confidence Interval $^{\mathrm{b}}$ & 0.961 to 0.994 \\
$\mathrm{z}$ statistic & 58.952 \\
Significance level P (Area $=0.5)$ & $<0.0001$ \\
\hline
\end{tabular}

aDeLong et al.8

'Binomial exact
Youden Index

\begin{tabular}{ll}
\hline Youden Index J & 0.9352 \\
$95 \%$ Confidence Interval $^{\mathrm{a}}$ & 0.8874 to 0.9693 \\
Associated Criterion & $>3.8$ \\
$95 \%$ Confidence Interval & $\mathrm{a}$ \\
Sensitivity & $>3.8$ to $>3.9$ \\
Specificity & 100 \\
\hline
\end{tabular}

aBCa bootstrap confidence interval (1000 iterations; random number seed: 978).

Comparison of ROC curves

\begin{tabular}{|c|c|c|c|}
\hline Variable 1 & \multicolumn{3}{|c|}{ O'sullivan test (mg/dl) } \\
\hline Variable 2 & \multicolumn{3}{|c|}{ Abdominal circumference $(\mathrm{cm})$} \\
\hline Variable 3 & \multicolumn{3}{|c|}{ Biparietal diameter $(\mathrm{cm})$} \\
\hline Variable 4 & \multicolumn{3}{|c|}{ Femur Length (cm) } \\
\hline Variable 5 & \multicolumn{3}{|c|}{ Expected fetal weight (gram) } \\
\hline Variable 6 & \multicolumn{3}{|c|}{ Abdominal subcutaneous tissue fat $(\mathrm{cm})$} \\
\hline Classification variable & \multicolumn{3}{|c|}{ OGTT Diagnosis } \\
\hline Sample size & \multicolumn{3}{|l|}{300} \\
\hline $\begin{array}{l}\text { Positive group: OGTT } \\
\text { Diagnosis }=1\end{array}$ & \multicolumn{3}{|l|}{7} \\
\hline \multirow{2}{*}{$\begin{array}{l}\text { Negative group: } \\
\text { OGTT Diagnosis }=0\end{array}$} & \multicolumn{3}{|l|}{293} \\
\hline & AUC & $\mathrm{SE}^{\mathrm{a}}$ & $95 \% \mathrm{CI}^{\mathrm{b}}$ \\
\hline Osullivan_test & 0.956 & 0.0404 & 0.926 to 0.976 \\
\hline abdominal_circumference & 0.878 & 0.0615 & 0.836 to 0.913 \\
\hline Biparietal_diameter & 0.927 & 0.0431 & 0.891 to 0.954 \\
\hline Femur_Length & 0.902 & 0.0457 & 0.863 to 0.933 \\
\hline expected_fetal_weight & 0.902 & 0.0511 & 0.863 to 0.934 \\
\hline $\begin{array}{l}\text { Abdominal_subcutan_tissue_ } \\
\text { fat }\end{array}$ & 0.983 & 0.00819 & 0.961 to 0.994 \\
\hline
\end{tabular}

\section{Diagnostic test accuracy statistics}

According to our results O'Sullivan test is a statistically significant discriminator of occurrence of gestational Diabetes mellitus with Area under the ROC curve (AUC) $=0.956$ (95\% Confidence interval $(\mathrm{CI})=0.926$ to 0.976$)$ (Zstatistics $=11.274$, Significance level $\mathrm{p}<0.0001$ ). This diagnostic criterion using Youden index is the level of $>179 \mathrm{mg} / \mathrm{dl}$ with a sensitivity of $85.71 \%$, specificity of $99.32 \%$, Positive predictive value (PPV) of $75 \%$ and negative predictive value of $99.7 \%$. Abdominal circumference is a statistically significant discriminator of occurrence of gestational Diabetes mellitus with Area under the ROC curve (AUC) $=0.878$. (95\% Confidence 
interval $(\mathrm{CI})=0.836$ to 0.913 ) (Zstatistics $=6.148$, Significance level $\mathrm{p}<0.0001)$. This diagnostic criterion using Youden index is the level of $>20.2 \mathrm{~cm}$ with a sensitivity of $100.00 \%$, specificity of $65.87 \%$, Positive predictive value (PPV) of $6.5 \%$ and negative predictive value of $100 \%$. Biparietal diameter is a statistically significant discriminator of occurrence of gestational Diabetes mellitus with Area under the ROC curve (AUC) $=0.927 \quad(95 \%$ Confidence interval $(\mathrm{CI})=0.891$ to 0.954$)$ (Zstatistics $=9.894$, Significance level $\mathrm{p}<0.0001$ ). This diagnostic criterion using Youden index is the level of $>6.24 \mathrm{~cm}$ with a sensitivity of $85.71 \%$, specificity of $99.2 \%$, Positive predictive value (PPV) of $10.6 \%$ and negative predictive value of $99.7 \%$.

Pairwise comparison of ROC curves

\begin{tabular}{ll}
\hline Osullivan_Test $\sim$ Abdominal_Circumference \\
\hline Difference between areas & 0.0775 \\
Standard Error ${ }^{\mathrm{c}}$ & 0.0836 \\
$95 \%$ Confidence Interval & -0.0863 to 0.241 \\
Z statistic & 0.927 \\
Significance level & $\mathrm{P}=0.3537$
\end{tabular}

Osullivan_Test $\sim$ Biparietal_Diameter

$\begin{array}{ll}\text { Difference between Areas } & 0.029 \\ \text { Standard Error } & \\ \text { 95\% Confidence Interval } & 0.0662 \\ \text { Z statistic } & -0.101 \text { to } 0.159 \\ \text { Significance level } & 0.438 \\ \end{array}$

Osullivan_Test $\sim$ Femur_Length

Difference between Areas

0.0539

Standard Error ${ }^{c}$

0.0699

95\% Confidence Interval

-0.0832 to 0.191

z statistic

0.77

Significance level

$\mathrm{P}=0.4410$

Osullivan_Test $\sim$ Expected_Fetal_Weight

Difference between Areas $\quad 0.0534$

$\begin{array}{ll}\text { Standard Error } & \\ & 0.0735\end{array}$

$95 \%$ Confidence Interval $\quad-0.0907$ to 0.197

$\begin{array}{ll}\text { z statistic } & 0.726\end{array}$

Significance level $\quad \mathrm{P}=0.4677$

Osullivan_Test $\sim$ Abdominal_Subcutan_Tissue_Fat

Difference between Areas

0.0271

Standard Error ${ }^{\mathrm{c}}$

0.0437

95\% Confidence Interval

-0.0586 to 0.113 z statistic $\quad 0.619$

Significance level

$\mathrm{P}=0.5357$

Abdominal_Circumference $\sim$ Biparietal_Diameter

Difference between Areas $\quad 0.0485$

Standard Error ${ }^{\mathrm{c}} \quad 0.0289$

95\% Confidence Interval $\quad-0.00817$ to 0.105

$\begin{array}{ll}\mathrm{z} \text { statistic } & 1.678\end{array}$

Significance level $\quad \mathrm{P}=0.0934$

Abdominal_Circumference $\sim$ Femur_Length

Difference between Areas $\quad 0.0236$

Standard Error ${ }^{\mathrm{c}} \quad 0.0182$

$95 \%$ Confidence Interval $\quad-0.0120$ to 0.0593

z statistic $\quad 1.299$

Significance level $\quad \mathrm{P}=0.1939$

Abdominal_Circumference $\sim$ Expected_Fetal_Weight

Difference between Areas $\quad 0.0241$

$\begin{array}{ll}\text { Standard Error }^{\mathrm{c}} & 0.017\end{array}$

$95 \%$ Confidence Interval $\quad-0.00922$ to 0.0575

$\mathrm{z}$ statistic $\quad 1.418$

Significance level $\quad \mathrm{P}=0.1561$

Abdominal_Circumference $\sim$ Abdominal_Subcutan_Tissue_Fat

Difference between Areas $\quad 0.105$

$\begin{array}{ll}\text { Standard Error } & \\ & 0.0579\end{array}$

$95 \%$ Confidence Interval $\quad-0.00895$ to 0.218

$\begin{array}{ll}\mathrm{z} \text { statistic } & 1.806\end{array}$

Significance level $\quad \mathrm{P}=0.0710$

Biparietal_Diameter $\sim$ Femur_Length

Difference between Areas $\quad 0.0249$

$\begin{array}{ll}\text { Standard Error } & 0.0181\end{array}$

$95 \%$ Confidence Interval $\quad-0.0106$ to 0.0603

$\begin{array}{ll}\text { z statistic } & 1.376\end{array}$

Significance level $\quad \mathrm{P}=0.1689$

Biparietal_Diameter $\sim$ Expected_Fetal_Weight

Difference between Areas $\quad 0.0244$

Standard Error

$95 \%$ Confidence Interval $\quad-0.00238$ to 0.0511 
Z statistic

1.786

Significance level

$\mathrm{P}=0.0741$

Biparietal_Diameter $\sim$ Abdominal_Subcutan_Tissue_Fat

Difference between Areas 0.0561

Standard Error

0.0383

95\% Confidence Interval

-0.0189 to 0.131

z statistic

1.465

Significance level

$\mathrm{P}=0.1429$

Femur_Length $\sim$ Expected_Fetal_Weight

Difference between Areas

0.000488

Standard Error

0.0139

95\% Confidence Interval

-0.0267 to 0.0276

z statistic

0.0352

Significance level

$\mathrm{P}=0.9719$

Femur_Length $\sim$ Abdominal_Subcutan_Tissue_Fat

Difference between Areas

0.0809

Standard Error

0.0421

95\% Confidence Interval

-0.00156 to 0.163

Z statistic

1.923

Significance level

$\mathrm{P}=0.0545$

Expected_Fetal_Weight $\sim$ Abdominal_Subcutan_Tissue_Fat

Difference between Areas

0.0804

Standard Error

0.0467

95\% Confidence Interval

-0.0111 to 0.172

z statistic

1.723

Significance level

$\mathrm{P}=0.0850$

${ }^{c}$ DeLong et al. ${ }^{8}$

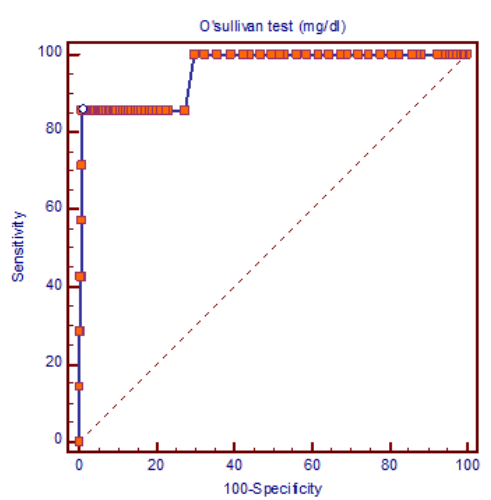

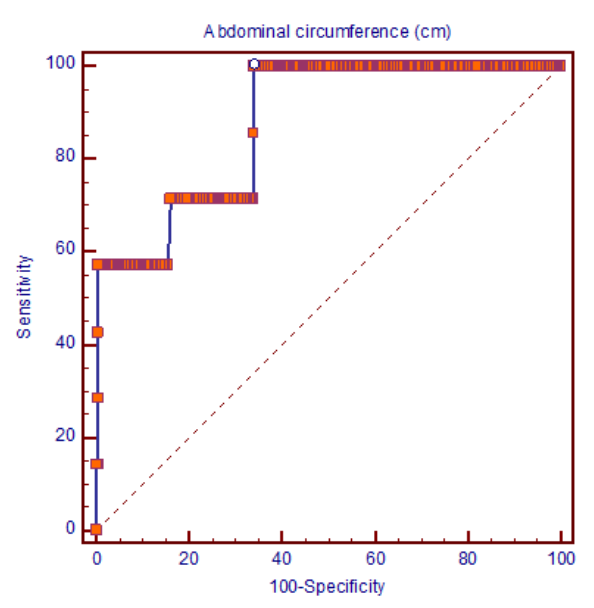

Figure I I Abdominal circumference sensitivity and specificity.

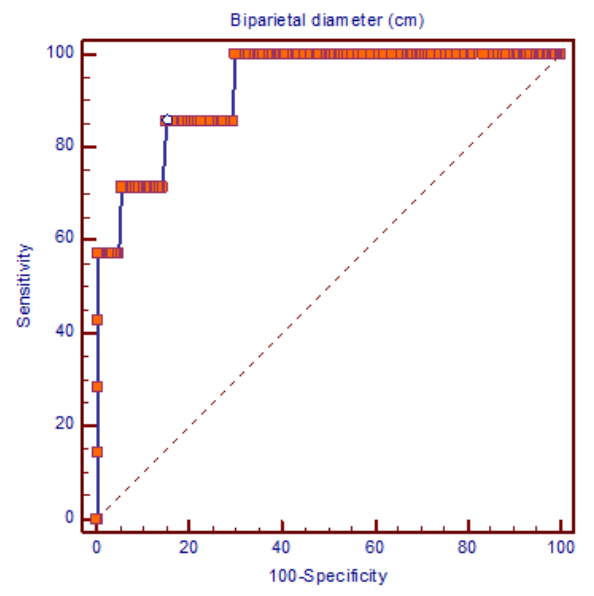

Figure I 2 Biparietal Diameter sensitivity and specificity.

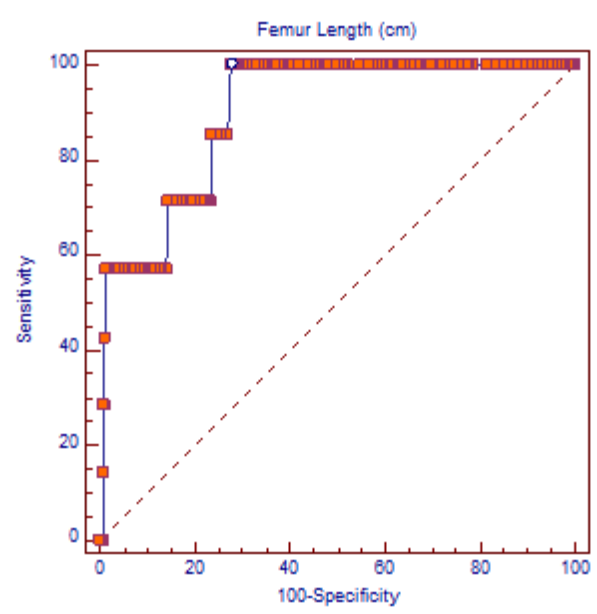

Figure I 3 Femur length sensitivity and specificity.

Figure 10 O' Sullivan test specificity and sensitivity. 


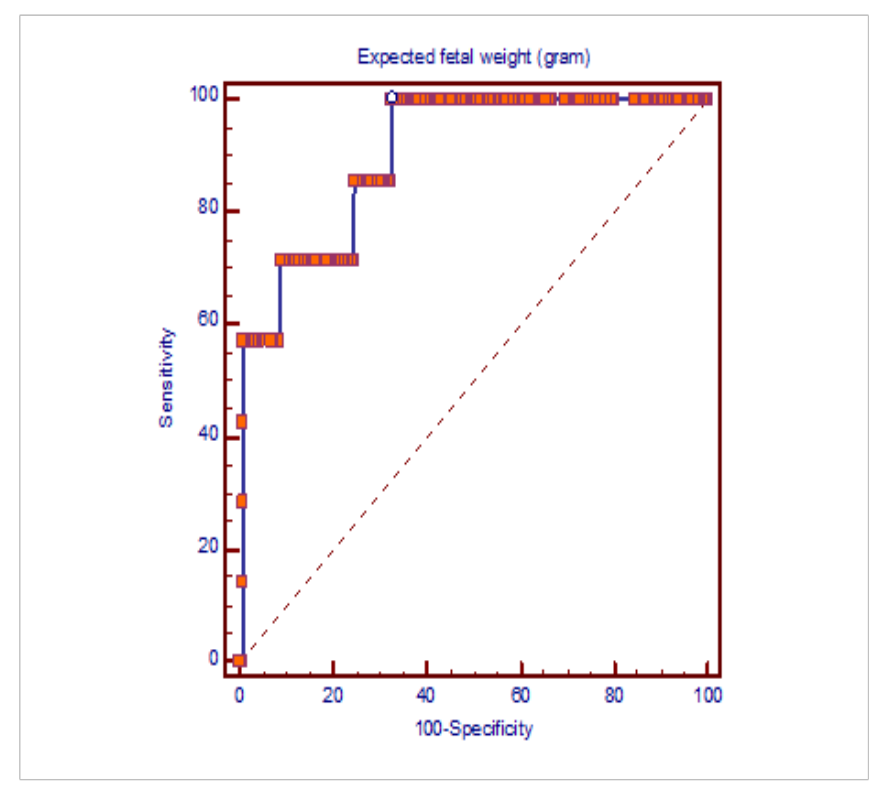

Figure I4 Expected fetal weight sensitivity and specificity.

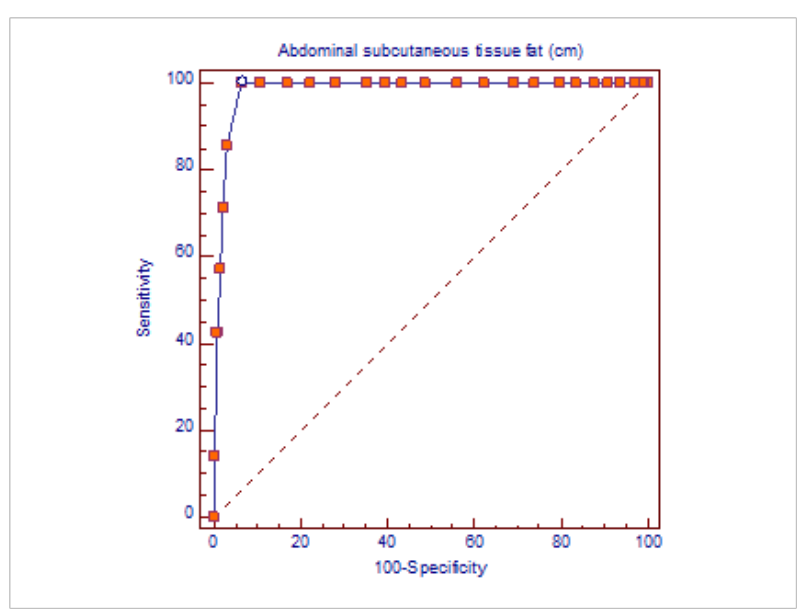

Figure 15 Abdominal subcutaneous fat sensitivity and specificity.

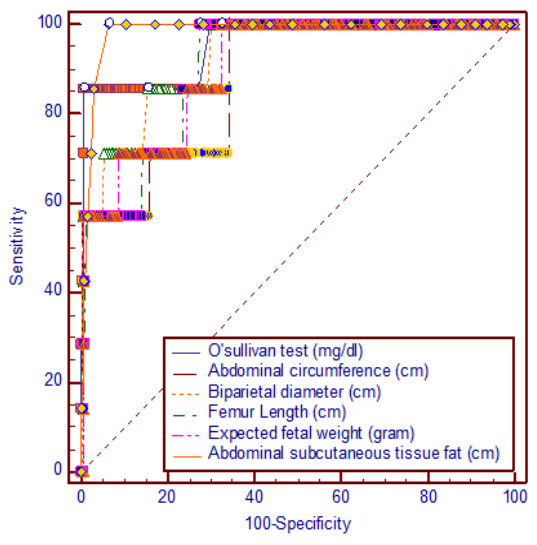

Figure 16 sensitivity and specificity for ultrasonographic parameters.
Femur length is a statistically significant discriminator of occurrence of gestational Diabetes mellitus with Area under the ROC curve $(\mathrm{AUC})=0.902(95 \%$ Confidence interval $(\mathrm{CI})=0.863$ to 0.933$)$ (Zstatistics $=8.795$, Significance level $\mathrm{p}<0.0001$ ). This diagnostic criterion using Youden index is the level of $>4.46 \mathrm{~cm}$ with a sensitivity of $100 \%$, specificity of $72.35 \%$, Positive predictive value (PPV) of $8 \%$ and negative predictive value of $100 \%$. Expected fetal weight is a statistically significant discriminator of occurrence of gestational Diabetes mellitus with Area under the ROC curve (AUC) $=0.902$ (95\% Confidence interval $(\mathrm{CI})=0.863$ to 0.934 ) (Zstatistics $=7.876$, Significance level $\mathrm{p}<0.0001)$. This diagnostic criterion using Youden index is the level of $>712 \mathrm{gm}$ with a sensitivity of $100 \%$, specificity of $67.58 \%$, Positive predictive value (PPV) of $6.9 \%$ and negative predictive value of $100 \%$. Abdominal subcutaneous tissue fat is a statistically significant discriminator of occurrence of gestational Diabetes mellitus with Area under the ROC curve (AUC) $=0.983$ (95\% Confidence interval $(\mathrm{CI})=0.961$ to 0.994$)$ (Zstatistics $=58.952$, Significance level $\mathrm{p}<0.0001)$. This diagnostic criterion using Youden index is the level of $>3.8 \mathrm{~cm}$ with a sensitivity of $100 \%$, specificity of $93.52 \%$, Positive predictive value (PPV) of $26.9 \%$ and negative predictive value of $100 \%$.

\section{Discussion}

Pregnancy is a state of insulin resistance revealing subclinical defect(s) in carbohydrate metabolism that may develop into a state of carbohydrate intolerance, or gestational Diabetes Mellitus. Therefore, pregnant women must be screened for gestational Diabetes Mellitus. Uniform diagnostic criteria are lacking for more than 40years. Our study aimed at assessing O'Sullivan test (50gram Glucose challenge test) against ultrasound measurements of abdominal circumference, biparital diameter, femur length, estimated fetal weight and abdominal subcutaneous skin fat, fetal liver length as screening for gestational diabetes mellitus between 24 and 28 weeks of gestation. The 2 hours OGTT was positive in only seven cases out of the 300 screened cases. Those positive cases were preliminary tested positive using O'sullivan test (statistically significant). In contrast, Acharya et al. ${ }^{10}$ studied the role of O'Sullivan test in screening of pregnant women at 24-36weeks for Gestational Diabetes. One Thousand cases were enrolled, of which only 8 cases $(0.8 \%)$ were screen positive in contrast to 60 to 63 cases per 1000 in most world series. Akram et al. ${ }^{11}$ used GCT to screen 1000 patients with analysis of risk factors to compare the efficiency of glucose challenge test with oral glucose tolerance test for detection of gestational diabetes, 450 patients were screened with positive result and 550 patients were screened negative. Out of 450 patients with positive GCT, OGTT detected $40 \%$ patients with true positive and 50 patients with false positive results. Out of $550 \mathrm{GCT}$ negative, OGTT screened 510 patients with true negative and 40 patients with false negative. So by performing OGTT 440 patients were diagnosed as gestational diabetics, while $56 \%$ had no diabetes. These results matched with ours as regards O' Sullivan test.

A range of ultrasound anthropometric parameters are used to determine normal fetal growth. In the present study we used ROC analysis to make a decision regarding fetal ultrasound parameters in predicting GDM. The present study has demonstrated that there is a highly significant correlation between fetal biparietal diameter, abdominal circumference, femur length, fetal weight, Abdominal subcutaneous tissue fat and blood glucose values during an OGTT in patients with GDM. The nature of the relationship detected implies that these parameters may be strong predictive factors for OGTT values; this was confirmed by ROC analysis. Conversely, no such relationship 
was found in the control group. Abdominal subcutaneous tissue fat was found to be a statistically significant discriminator of occurrence of gestational Diabetes mellitus with Area under the ROC curve (AUC) 0.983 . with a sensitivity of $100 \%$, specificity of $93.52 \%$ with regard to a cut-off value of $>3.8 \mathrm{~cm}$, Positive predictive value (PPV) of $26.9 \%$ and negative predictive value of $100 \%$. One of the major disadvantages of many of the screening tests used currently is falsepositive results. In the present study, Abdominal subcutaneous tissue fat measurements demonstrated a specificity near that for glucose tolerance test results based on data in the literature. Recently, studies have shown that abdominal subcutaneous tissue fat measurements, either on their own or incorporated into conventional fetal weight prediction formulae, could be used to evaluate fetal growth, and, in addition, assess whether maternal glucose levels are normal. ${ }^{12}$

In a comparison of abdominal subcutaneous tissue fat thickness (ASCTT) between fetuses from a group of mothers with gestational diabetes and those from a normal control group, there were significant differences between initial fetal ASCTT, but no difference after the mothers had been treated for diabetes. The measurement of fetal ASCTT gives a more accurate estimation of the stability of maternal glucose levels than a maternal ambulatory glycemic profile. ${ }^{12}$ As reported, ultrasound measurements of subcutaneous adipose tissue may be a reliable indicator of the fetal metabolic state in pregnancies with GDM. ${ }^{12}$ Meanwhile, the availability of reference values for fetal subcutaneous fat ultra-sound measurements may provide clinically useful information to identify excessive fetal fat deposition and evaluate maternal glucose levels in pregnancies with GDM, beside the routine fetal ultrasonographic biometric parameters. Comparing our results with similar studies, Vedavathi et al. ${ }^{13}$ studied 30 cases with an established diagnosis of gestational diabetes at 24-28weeks of gestation, by measuring abdominal circumference, head circumference and the estimated fetal weight. Abdominal circumferences (AC) of $27(90 \%)$ cases, Head circumferences (HC) of $20(67 \%)$ cases and estimated fetal weight (EFW) of $8(27 \%)$ cases were $>90$ percentile. He concluded that fetal growth parameters show significant high values in GDM. These results are criticized by the very small sample he worked upon.

In another large prospective cohort, Smith et al., ${ }^{14}$ concluded that excessive fetal growth preceded the diagnosis of gestational diabetes, by analysing data from 4069 pregnant women. All women provided blood samples and underwent ultrasounds at 20,28 and 36weeks of gestation, as well as an oral glucose tolerance test at 28 weeks. Risks were set for abdominal circumference $>90^{\text {th }}$ percentile and $\mathrm{HC} / \mathrm{AC}$ ratio $<10^{\text {th }}$ percentile. Within the cohort, 171 women $(4.2 \%)$ were diagnosed with gestational diabetes at 28 weeks. Researchers found no association between fetal measurements at 20 weeks of gestation and a subsequent diagnosis of gestational diabetes; however, at 28 weeks, researchers found an increased risk for increased fetal abdominal circumference $(\mathrm{RR}=2.05 ; 95 \% \mathrm{CI}, 1.37-3.07)$ and decreased head circumference to abdominal circumference ratio $(\mathrm{RR}=1.97$; $95 \% \mathrm{CI}$, 1.3-2.99) in mothers with gestational diabetes. At 28 weeks, mothers with gestational diabetes had a nearly fivefold increased risk for greater fetal abdominal circumference $(\mathrm{RR}=4.52 ; 95 \% \mathrm{CI}, 2.98-6.85)$ and a nearly threefold increased risk for decreased head circumference to abdominal circumference ratio $(\mathrm{RR}=2.8 ; 95 \% \mathrm{CI}, 1.65-4.78)$. Researchers also found that increased fetal abdominal circumference was associated with a nearly fourfold increased risk for the fetus being born large for gestational age $(\mathrm{RR}=3.86 ; 95 \% \mathrm{CI}, 2.37-6.29) .{ }^{14}$

Honestly we cannot criticize these results but we find them more logic than ours as the sample size was bigger and the cases were preliminary diagnosed as having gestational diabetes mellitus. Bulhing et al., ${ }^{15}$ studied the relationship between sonographically estimated fetal subcutaneous adipose tissue measurements and neonatal skin fold measurements in diabetic fetopathy, but his study was conducted at 37 weeks of gestation. Bulhing enrolled 172 patients (142 controls and 30 with gestational diabetes), both fetal subcutaneous fat tissue and estimated fetal weight were measured. He concluded that ultrasound examination is a reliable method for noninvasive intrauterine measurement of fetal subcutaneous tissue for predicting mechanical neonatal skinfold thickness measurements and thus gestational diabetes. Our results matched with those studies where a positive correlation was detected between fetal ultrasonographic biometric parameters including ASCTT and GDM, but we failed to find a positive correlation between fetal liver length and GDM although maternal hyperglycemia is related to fetal hyperglycemia and hyperinsulinemia, which has a significant impact on the growth of insulin-dependent tissues and organs, such as the liver. ${ }^{15}$

\section{Conclusion}

i. Screening for gestational diabetes by ultrasound measurement of abdominal circumference, biparietal diameter and estimated fetal weight between 24-28weeks of gestation is a reliable, cost-effective method that can be used solely or combined with O'Sullivan test.

ii. The addition of fetal abdominal subcutaneous fat layer measurement (using a cut-off value $\geq 3.8 \mathrm{~mm}$ ) yielded better accuracy, better positive and negative predictive value, better sensitivity and specificity.

\section{Acknowledgements}

None.

\section{References}

1. Chen L, Wu JJ, Chen XH, et al. Measurement of fetal abdominal and subcapsular subcutaneous tissue thickness during pregnancy to predict macrosomia: A pilot study. PLOS One. 2014;9(3):e93077.

2. Wier LM, Witt E, Burgess J, et al. Hospitalizations related to diabetes in pregnancy, 2008. HCUP Statistical Brief \#102. Agency for Healthcare Research and Quality. USA: Agency for Healthcare Research and Quality; 2010. p. 1-10.

3. American Diabetes Association. Standards of medical care in diabetes. Diabetes Care. 2014;37(suppl 1):S14-80.

4. Khoury JC, Dolan LM, Vandyke R, et al. Fetal development in women with diabetes: Imprinting for a life-time. J Matern Fetal Neonatal Med. 2012;25(1):11-14

5. IBM SPSS Statistics for Windows, Version 21.0. USA: Armonk; 2012.

6. Field A. Discovering Statistics Using SPSS. 2nd ed. USA: SAGE Publications Ltd; 2006.

7. Mann HB, Whitney DR. On a test of whether one of two random variables is stochastically larger than the other. Annals of Mathematical Statistics. 1947;18(1):50-60.

8. DeLong ER, DeLong DM, Clarke-Pearson DL. Comparing the areas under two or more correlated receiver operating characteristic curves: a nonparametric approach. Biometrics. 1988;44(3):837-845.

9. MedCalc Statistical Software version 14.8.1 (MedCalc Software bvba, Ostend, Belgium; 2014 http://www.medcalc.org). 
10. Acharya N, Inamdar A, Inamdar S. Role of O Sullivan S test in screening of pregnant women for gestational diabetes in rural area. JSAFOG. 2011;3(2):86-88.

11. Akram N, Butt F. Universal screening with glucose challenge test in detection of gestational diabetes. Biomedica. 2014;3(1):29-33.

12. Chen L, Wu JJ, Chen XH, et al. Measurement of fetal abdominal and subscapular subcutaneous tissue thickness during pregnancy to predict macrosomia: a pilot study. PLoS One. 2014;9(3):e93077.
13. Vedavathi KJ, Swamy RM, Shekharappa KP, et al. Influence of Gestational Diabetes Mellitus on Fetal growth parameters. Int $J$ Biol Med Res. 2011;2(3):832-834.

14. Sovio U, Murphy HR, Smith GC. Accelerated fetal growth prior to diagnosis of gestational diabetes mellitus: a prospective cohort study of nulliparous women. Diabetes Care. 2016;39(6):982-987.

15. Buhling KJ, Doll I, Siebert G, et al. Relationship between sonographically estimated fetal subcutaneous adipose tissue measurements and neonatal skinfold measurements. Ultrasound Obstet Gynecol. 2012;39:558-562. 